\title{
Neural Network Approach for Underwater Acoustic Communication in the Shallow Water
}

\author{
K.C. Park, J.R. Yoon \\ Department of Information and Communications Engineering \\ Pukyong National University, Korea
}

\begin{abstract}
The transmitted acoustic signals are severely influenced by boundaries like as sea surface and bottom in the shallow water. Very large reflection signals from boundaries cause inter-symbol interference effect, the performance of the communication are degraded. Usually, to compensate the reflected signals under this kind of acoustic channel, is adopting the channel estimation based equalizers. In this study, we express neural network approaches for image data transmission in the shallow water. A simple neural network is adopted for the decision from output data. The QPSK system is used for the underwater acoustic communication simulations and experiments.
\end{abstract}

Keywords- underwater acoustic communication; shallow water; QPSK system; neural network; inter-symbol interference

\section{INTRODUCTION}

The underwater acoustic communication channel is known to exhibit a frequency selective channel by multi-path delay spread in a multipath channel such as shallow water. The performance of the underwater acoustic communication system degrades due to inter-symbol interference (ISI) by multi-reflections from the boundaries.[1-3] The channel estimation based equalizers are open adopted to compensate these phenomena. This paper presents techniques using the neural network to send image data with less error. The simple neural network is used for some numerical experiments in simulation signals and experimental signals. The QPSK system is used for the underwater acoustic communications simulations.

\section{CONFIGURATION FOR SIMULATION AND EXPERIMENT}

There are two channels - I channel and Q channel - in Quadrature phase-shift keying (QPSK) system shown as Figure I . The transmitted signal is demodulated separately to two output signals using cosine signal or sine signal with same carrier frequency of modulation system, and then output signals are converted to 4 states data [00 0110 11] from each output signal. It means the numbers of the input and output signal's types are 4 when neural network is applied in this system.

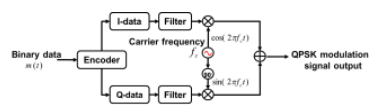

(a)

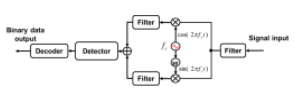

(b)

(a) modulation system and (b) demodulation system.

FIGURE I . QPSK SYSTEM.

Figure II shows the configuration of sea experiment and its sound velocity profile in shallow water located in the George island near to Busan city in Korea. The range between transmitter and receiver is set to be 100 , or $400 \mathrm{~m}$. The depths of receiver and transmitter are set to be 7 and $10 \mathrm{~m}$, respectively. The channel characteristics for the numerical simulation are obtained as figure III, from this environment parameter as shown figure II. We assumed that the channel impulse response had 5 signals as shown eqn. (1) - direct, bottom reflected, surface reflected, bottom-surface reflected, and surface - bottom reflected signals.

$$
h(t)=\sum_{n=0}^{N} a_{n} \delta\left(t-\tau_{n}\right)
$$

Here, ${ }^{a_{n}}$ is the nth multipath signal's amplitude, ${ }^{\tau_{n}}$ is the nth multipath delay time, and $\mathrm{N}$ is chosen by 4 . In order to understand the characteristics of the each channel, the effective delay spread $\tau_{r m s}$ and the coherence bandwidth $W$ are calculated using eqns.(2)-(4). The $\tau_{r m s}$ is given as[3, 4]

$$
\tau_{r m s}=\sqrt{\overline{\tau^{2}}-(\bar{\tau})^{2}} .
$$
by

Here, the average delays $\overline{\tau^{2}}$ and $\bar{\tau}$ are respectively given

$$
\overline{\tau^{2}}=\frac{\sum_{n} a_{n}^{2} \tau_{n}^{2}}{\sum_{n} a_{n}^{2}}, \quad \bar{\tau}=\frac{\sum_{n} a_{n}^{2} \tau_{n}}{\sum_{n} a_{n}^{2}} .
$$

The relationship between the effective delay spread and the channel's coherence bandwidth $W$ is given by

$$
W \approx \frac{1}{5 \tau_{\text {rms }}}
$$

If the channel's coherence bandwidth is less than that of the signals, it is difficult to send data without errors due to the channel distortion. 
The transmission rates are chosen by 100, 400, and 1600 symbols per second (sps). The image for the transmission is the standard Lenna image consists of 35x35 pixels with 8 bits per pixel, which amounts to 9,800 bits of data. The carrier frequency and sampling frequency are chosen by $16 \mathrm{kHz}$ and $128 \mathrm{kHz}$, respectively.

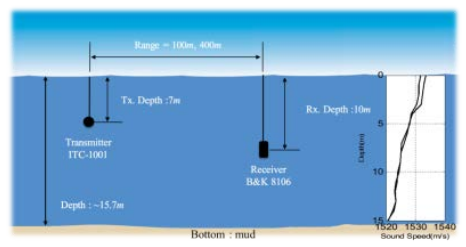

FIGURE II . EXPERIMENTAL CONFIGURATION.
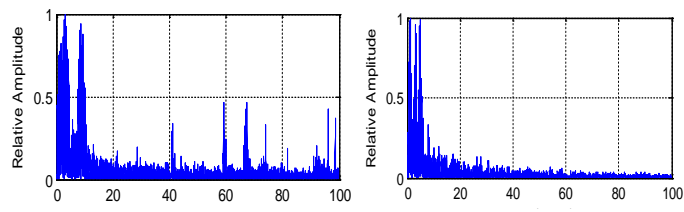

(a)
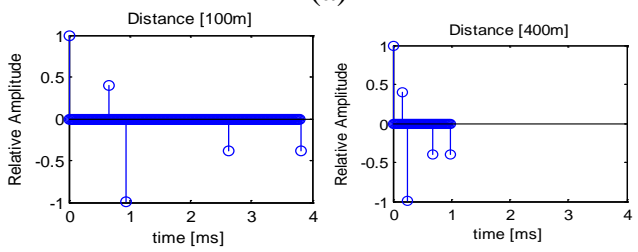

(b)

(a) experimental, (b) simulation

FIGUREIII. CHANNELS’ IMPULSE RESPONSES.

At next, the used neural network is shown as figureIV. It consists of an input layer, a hidden layer, and an output layer. The expected results of the output layer are [1000 01000010 0001] at output $\mathrm{A}(1) \sim \mathrm{A}(4)$ that are corresponding to the output of QPSK systems to be 4 stats [00 0110 11]. In the neural network system with the hidden layer, the weightings are obtained by the learning with some learning datum in the input layer and the expected output in the output layer. The first some part of the QPSK demodulated signal is used for the input signal. The input layer consists of 2 , 4, or 8neurons, the hidden layer and the output layer is 20 neurons and 4 neurons, respectively.[5]

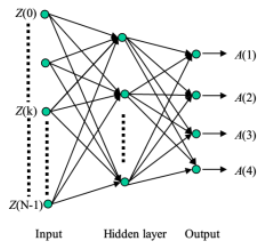

FIGURE IV. NEURAL NETWORK DIAGRAM FOR QPSK SYSTEM.

\section{RESULTS AND DISCUSSION}

From eqn. (4), the calculated channel coherence bandwidths $W$ are about 199.32 and $760.63 \mathrm{~Hz}$ at distance 100 and $400 \mathrm{~m}$, respectively. This result is used to select the number of the input layer. For example, the number of the input layer for the $100 \mathrm{~m} \& 400$ spouse is 4 (=400 sps / 199.32 $\mathrm{Hz} * 2$ bit).

Table I shows the numerical simulation results without any compensation in the QPSK communication system. The training iterations are chosen to be $16,16 * 8$, or $16 * 8 * 4$ according to the number of input layer -2 , 4 , or 8 . The parts on right upper triangle (Bold style) belong to the frequency non-selective fading channel - bandwidth of the signal is less than that of channel, the others belong to the frequency selective channel. The errors are owing to the phase distortion by the reflected waves in the frequency non-selective fading channels, it needs some compensation procedures.

TABLE I . SIMULATION RESULTS WITHOUT ANY COMPENSATION.

\begin{tabular}{|c|c|c|}
\hline $\begin{array}{c}\text { distance } \\
\text { sps }\end{array}$ & $100 \mathrm{~m}$ & $400 \mathrm{~m}$ \\
\hline $100 \mathrm{sps}$ & $\mathbf{0 . 5 0 0}$ & $\mathbf{0 . 1 3 7}$ \\
\hline $400 \mathrm{sps}$ & 0.429 & $\mathbf{0 . 1 3 7}$ \\
\hline $1600 \mathrm{sps}$ & 0.196 & 0.198 \\
\hline
\end{tabular}

Table II shows the results using neural network in simulation. All results are zero error in frequency nonselective channel. Also, using enhancement of the number of input layer, bold style's results show good results like as the channel would be frequency non-selective fading.

TABLE II . SIMULATION RESULTS WITH NEURAL NETWORK.

\begin{tabular}{|c|c|c|c|c|c|c|}
\hline $\begin{array}{c}\text { distance } \\
\text { sps }\end{array}$ & \multicolumn{3}{|c|}{$100 \mathrm{~m}$} & \multicolumn{3}{c|}{$400 \mathrm{~m}$} \\
\hline $\begin{array}{c}\text { No. of } \\
\text { input } \\
\text { layer }\end{array}$ & 2 & 4 & 8 & 2 & 4 & 8 \\
\hline 100 sps & 0.000 & 0.000 & 0.000 & 0.000 & 0.000 & 0.000 \\
\hline 400 sps & 0.175 & $\mathbf{0 . 0 1 8}$ & $\mathbf{0 . 0 0 0}$ & 0.000 & 0.000 & 0.000 \\
\hline $\begin{array}{c}1600 \\
\text { sps }\end{array}$ & 0.245 & 0.154 & $\mathbf{0 . 0 1 6}$ & 0.123 & $\mathbf{0 . 0 0 9}$ & $\mathbf{0 . 0 3 6}$ \\
\hline
\end{tabular}

Table III shows the results using neural networks with experiment data. In all results, the input layer consists of 8 neurons, the hidden layer and the output layer is 30 neurons and 4 neurons, respectively. In experimental data, the performance with neural network shows little bit better performance than those of without neural network.

TABLEIII. EXPERIMENT RESULTS WITH NEURAL NETWORK.

\begin{tabular}{|l|c|c|c|c|}
\hline $\begin{array}{l}\text { distance } \\
\text { sps }\end{array}$ & \multicolumn{2}{|c|}{$100 \mathrm{~m}$} & \multicolumn{2}{c|}{$400 \mathrm{~m}$} \\
\hline & $\begin{array}{c}\text { w/o. } \\
\text { NN }\end{array}$ & $\begin{array}{c}\text { w. } \\
\text { NN }\end{array}$ & $\begin{array}{c}\text { w/o. } \\
\text { NN }\end{array}$ & $\begin{array}{c}\text { w. } \\
\text { NN }\end{array}$ \\
\hline $100 \mathrm{sps}$ & 0.291 & 0.220 & 0.204 & 0.140 \\
\hline $400 \mathrm{sps}$ & 0.234 & 0.230 & 0.116 & 0.084 \\
\hline
\end{tabular}

\section{CONCLUSION}

From all the above results, in the frequency non-selective fading channel, it is enough to get better results with neural network in numerical simulations. Chosen to be the number of input layer according to channel's bandwidth is also significant. In experimental data, the performance with neural network show little bit better performance than those of 
without neural network. This difference between simulation and experiment are shown that the results caused by varying experimental environment, unlike the time-invariant simulation environment.

\section{ACKNOWLEDGMENT}

This work was supported by a Research Grant of Pukyong National University(2013 year).

\section{REFERENCES}

[1] J. Park, J. R. Yoon, and J. Park, Frequency and Temporal Coherence Variation for Sea Surface Fluctuation, Jpn. J. Appl. Phys. 48, pp. 07GL03, 2009.

[2] J. Park, K. Park, and J. R. Yoon, Underwater Acoustic Communication Channel Simulator for Flat Fading, Jpn. J. Appl. Phys. 49, pp. 07HG10, 2010.

[3] J. Kim, K. Park, J. Park, and J. R. Yoon, Coherence Bandwidth Effects on Underwater Image Transmission in Multipath Channel, Jpn. J. Appl. Phys. 50, pp. 07HG05, 2011.

[4] Theodore S. Rappaport, Wireless Communications: Principles and Practice, 2nd Ed., Prentice Hall PTR: New Jersey, pp. 400, 2002.

[5] J.-S. R. Jang, C.-T. Sun \& E. Mizutani, Neuro-Fuzzy and Soft Computing, (Chapter III). Neural Networks, Prentice Hall: New Jersey, pp. 197-331, 1997. 